\title{
Study of the Orthoptic Assessment in Refractive Eye Surgery
}

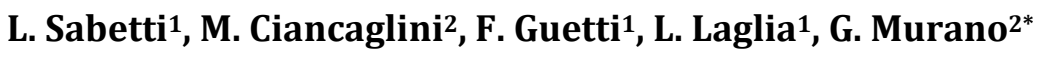 \\ ${ }^{1}$ Department of Biotechnological and Applied Clinical Sciences, University of L'Aquila, L'Aquila, Italy \\ ${ }^{2}$ Departmente of Life, Health \& Environmental Sciences, L’Aquila, Italy \\ Email: *gianlucamurano89@gmail.com
}

How to cite this paper: Sabetti, L., Ciancaglini, M., Guetti, F., Laglia, L. and Murano, G. (2020) Study of the Orthoptic Assessment in Refractive Eye Surgery. Open Journal of Ophthalmology, 10, 55-58.

https://doi.org/10.4236/ojoph.2020.101007

Received: November 20, 2019

Accepted: January 5, 2020

Published: January 8, 2020

Copyright $\odot 2020$ by author(s) and Scientific Research Publishing Inc. This work is licensed under the Creative Commons Attribution International License (CC BY 4.0).

http://creativecommons.org/licenses/by/4.0/

\begin{abstract}
Purpose: The aim of our study was to assess the variations in fusion and stereopsis before and after refractive surgery. Methods. We conducted a retrospective study. 140 patients ( $78 \mathrm{M}, 62 \mathrm{~F}$ ) were selected, aged 20 - 59 years (mean age $36 \pm 10$ DS). All patients received a comprehensive ophtalmological and orthoptic examination. Surgery was performed using a MEL-80 excimer laser (Carl Zeiss Meditec, Jena, Germany). Results. Fusional convergence amplitudes after refractive eye surgery range from at near $18-20$ PD in $42(30 \%)$ patients; 25 - $30 \mathrm{PD}$ in 56 (40\%) patients; 35 - $40 \mathrm{PD}$ in 42 (30\%) patients, at far 20 - $25 \mathrm{PD}$ in 84 (60\%) patients; 30 - $40 \mathrm{PD}$ in 56 (40\%) patients, fusional divergence at near after refractive eye surgery range from at near $6-8 \mathrm{PD}$ in $108(75.7 \%)$ patients; $10-12 \mathrm{PD}$ in 52 (37.1\%), at far 6 - 8 PD in $126(90 \%)$ patients; $10-12 \mathrm{PD}$ in $14(10 \%)$ patients. None of the patients developed any ocular deviations. NCP, on average, decreases from $9.4 \pm 1.5 \mathrm{~cm}$ to $9.1 \pm 0.9$ $\mathrm{cm}$ after. None of these patients with a normal NCP before surgery developed an abnormal NCP after refractive surgery. Eighteen patients (12.8\%) had a stereopsis higher than $60 \mathrm{~s}$ of arch before surgical intervention. Of these, in 2 cases $(2.8 \%)$ stereopsis increased from 200 to $40 \mathrm{~s}$ of arch after surgery. In the rest of patients stereopsis remained unchanged. Conclusion: The increase in fusion at near appears to be considerably interesting, whereas there is no worsening of stereopsis. A careful pre-surgery orthoptic evaluation is extremely revelant for a safe refractive surgery, this reducing the risk of complications associated with fusion and stereopsis.
\end{abstract}

\section{Keywords}

Orthoptic, Refractive, Excimer, Fusion, Stereopsis, Convergence

\section{Introduction}

Binocular vision describes the process by which the brain integrates the inputs 
from the two eyes to blend them into a single three-dimensional picture. Refractive surgery offers some advantages: neutralization of the prismatic effects, improvement in visual acuity, improvement in binocular vergence and fusion, strengthening or recovery of binocular vision.

In 2005 Sabetti et al. demonstrated how the refractive surgery allows the correction of accommodative esotropia and therefore without the modification of the forms of esotropia is not caused by an abnormal AC/A ratio [1]. Hashemi (2013) highlights hot the near poing of convergence and the near point of accommodation may increase significantly after photorefractive keratectomy (PRK) [2]. In 2014 Han reported an increase in the fusional vergence at near following refractive surgery [3]. Chung (2014) underlines the risk of a change in the ocular alignment, particulary in cases with a large-angle heterophoria/ heterotropia [4].

Aim of the study is to assess the changes in the binocular vision following a refractive surgery intervention through an exam of the main variations: accommodation, visual parallelism and fusional amplitudes.

\section{Methods}

We conducted a retrospective study. $\mathrm{N}^{\circ} 140$ patients $(78 \mathrm{M}, 62 \mathrm{~F})$ ranging in age from 20 - 59 years (280 eyes) were recruited for the study.

All patients in the study are selected patients to perform refractive surgery. Patients with too hight refractive defects compared to their corneal thickness were excluded; patients with keratoconus or suspected keratoconus were excluded; patients with abnormal Shirmer's test were excluded.

All patients underwent a complete ophthalmic examination: visual acuity, biomicroscopy, tonometry and exploration of the ocular fundus. Also, both topographical and pachymetric maps were performed.

All patient underwent complete orthoptic examination using corneoscleral contact lenses, measurement of near point of convergence (NCP) with punctiform source of light, measurement of the fusional amplitudes with Berens prisms and Lang's stereoscopic test II.

The surgery intervention was performed using the MEL-80 excimer laser (Carl Zeiss Meditec, Jena, Germany). MEL 80 is an excimer laser with a wide field of application, including Femto-LASIK, LASIK customized treatments ad PRSBYOND Laser Blended Vision and topography guided treatment, Surface ablation treatments as PRK, LASEK, therapeutic treatment as Phototherapeutic Keratectomy (PTK).

All patients gave their written consent to data processing.

\section{Results}

Pre- and post-operative measurements of visual acuity were not significantly different. Postoperatively 274 (97.8\%) eyes became emmetropic, hypercorrections within $0.5 \mathrm{D}$ were found in 6 eyes $(2.1 \%)$. 
Fusional convergence amplitudes at near before surgery range from:

- 16 - 18 PD in 42 (30\%) patients;

- 20 - 25 PD in 42 (30\%) patients;

- 30 - 35 PD in 42 (30\%) patients;

- 35 - 40 PD in 14 (10\%) patients.

Fusional convergence amplitudes at far before surgery range from:

- 16 - 18 PD in 56 (40\% patients; 20 - 25 PD in 42 (30\%) patients;

- 30 - 35 PD in 42 (30\%) patients.

Fusional convergence amplitudes at near after refractive eye surgery range from:

- 18 - 20 PD in 42 (30\%) patients;

- 25 - 30 PD 56 (40\%) patients;

- 35 - 40 PD in 42 (30\%) patients.

Fusional convergence amplitudes at far after refractive eye surgery range from:

- 20 - 25 PD in 84 (60\%) patients;

- 30 - $40 \mathrm{PD}$ in 56 (40\%) patients.

Fusional divergence at near before refractive eye surgery range from:

- 6 - 8 PD in 52 (37.1\%) patients;

- 10 - 12 PD in 88 (62.9\%) patients.

Fusional divergence at far before refractive surgery range from

- 6 - 8 PD in 88 (62.9\%) patients;

- $10-12 \mathrm{PD}$ in 52 (37.1\% patients).

Fusional divergence at near after refractive eye surgery range from:

- 6 - 8 PD in 108 (75.7\%) patients;

- 10 - 12 PD in 52 (37.1\%) patients.

Fusional divergence at near after refractive eye surgery range from

- 6 - 8 PD in 126 (90\%) patients;

- 10 - 12 PD in 14 (10\%) patients.

Fusional convergence amplitudes assessed after surgery, both at far and at near fixation range from:

- 20 - 25 PD in 40 (28.6\%) patients;

- 25 - $30 \mathrm{PD}$ in 70 (50\%) patients;

- 30 - $40 \mathrm{PD}$ in 30 (21.4\%) patients.

Divergent fusional amplitudes assessed after surgery, both at far and at near fixation, range from:

- 8 - 6 PD in 60(42.9\%) patients;

- 10 - 8 PD in 50 (35.7\%) patients;

- 12 - 10 PD in 30 (21.4\%) patients.

VISUAL AXIS ALIGNMENT: No patients developed ocular deviations.

The NCP (Near Point of Convergence), on average, decreases from $9.4 \pm 1.5$ $\mathrm{cm}$ to $9.1 \pm 0.9 \mathrm{~cm}$ following surgery. None of these patients with a normal NPC before surgery, developed an abnormal NPC following the refractive surgery. 
STEREOPSIS: Eighteen (12.8\%) patients presented a stereopsis worse than 60 seconds of arc before surgery. Of these, stereopsis improved from 200 to $40 \mathrm{sec}$ onds of arc prostoperatively in 4 cases. Both had hypermetropic astigmastims before surgery and became emmetropic following the procedure. Stereopsis remained unchanged in the remaining patients.

\section{Conclusions}

Refractive surgery does not induce ocular deviations in orthophoric subjects. An increase in the fusional convergence both at far and at near fixation was observed, whilst the fusional divergence did not record any significant change. The stereopsis test results did not detect any significant alteration. Evaluating the causes for diplopia following the refractive surgery, Kushner suggested five mechanism: technical problems, prior deviations, aniseikonia, iatrogenic monovision and improper control of accommodation.

With this study what we want to prove is that: the correct strabological assessment and clinical evaluation before refractive surgery are advisable and important.

\section{Conflicts of Interest}

The authors declare no conflicts of interest regarding the publication of this paper.

\section{References}

[1] Sabetti, L., Spadea, L., D’Alessandri, L. and Balestrazzi, E. (2005) Photorefractive Keratectomy and Laser in Situ Keratomileusis in Refractive Accommodative Esotropia. Journal of Cataract \& Refractive Surgery, 31, 1899-1903. https://doi.org/10.1016/j.jcrs.2005.03.077

[2] Hashemi, H., Samet, B., Mirzajani, A., Khabazkhoob, M., Rezvan, B. and Jafarzadehpur, E. (2013) Near Point of Accommodation and Convergence after Photorefractive Keratectomy (PRK) for Myopia. Binocular Vision and Strabology Quarterly, 28, 29-35.

[3] Han, J., Hong, S., Lee, S., Kim, J.K., Lee, H.K. and Han, S.H. (2014) Changes in Fusional Vergence Amplitudes after Laser Refractive Surgery for Moderate Myopia. Journal of Cataract \& Refractive Surgery, 40, 1670-1675. https://doi.org/10.1016/j.jcrs.2014.01.043

[4] Chung, S.A., Kim, W.K., Moon, J.W., Yang, H., Kim, J.K., Lee, S.B. and Lee, J.B. (2014) Impact of Laser Refractive Surgery on Ocular Alignment in Myopic Patients. Eye, 28, 1321-1327. https://doi.org/10.1038/eye.2014.209 